\title{
Field testing of a cyclist collision avoidance system for heavy goods vehicles
}

\author{
Yanbo Jia and David Cebon
}

\author{
Submitted to IEEE Transactions on Vehicular Technology: 30/6/2015 \\ Accepted for publication: 8/2/2016
}

\begin{abstract}
This research focused on preventing collisions between cyclists and heavy goods vehicles (HGVs). A collision avoidance system, designed to avoid side-to-side collisions between HGVs and cyclists, is proposed. The cyclist's motion relative to the HGV is measured with an array of ultrasonic sensors. The detected distances from ultrasonic sensors are processed in real time to construct a smooth trajectory for the cyclist. The controller assumes constant acceleration and constant yaw rate for both the HGV and the cyclist and extrapolates the relative motion forward in time. The HGVs' brakes are engaged if a collision is predicted. A prototype system was built and fitted onto a test truck. The proposed collision avoidance system was tested in real time and proved to be effective within certain speed ranges.
\end{abstract}

Index Terms - ultrasonic sensors, cyclist protection, collision avoidance, position prediction, emergency braking, heavy goods vehicles

\section{InTRODUCTION}

$\mathrm{H}$ EAVY goods vehicles (HGVs), defined as having a

Gross Combination Mass (GCM) of over 3.5 tons, transport over 90 per cent of all freight moved by road in the UK. This equated to 132 billion ton-kilometres in $2009,73 \%$ of which was carried by articulated vehicles [1]. HGVs are overrepresented in accidents and lead to a substantial proportion of casualties and fatalities on the road. According to a consultation report prepared by the Royal Society for Prevention of Accidents (RoSPA), 18.3 per cent of all road fatalities occur in accidents involving an $\mathrm{HGV}$, despite the fact that they only covered 5.8 per cent of the mileage of all vehicle traffic in the UK [2]. RoSPA reported that, although only 2 per cent of cyclists' accidents occurred in collisions

Paper submitted for review on Tuesday 30 June, 2015.

Yanbo Jia, Dr., is with Department of Engineering, University of Cambridge, Trumpington Street, Cambridge CB2 1PZ, UK (email: yj253@cam.ac.uk).

David Cebon, Prof., BE, PhD, FREng, FIMechE, is with Department of Engineering, University of Cambridge, Trumpington Street, Cambridge CB2 1PZ, UK (e-mail: dc@eng.cam.ac.uk)

Corresponding author: David Cebon (email: dc@eng.cam.ac.uk). with HGVs, 22 per cent of them resulted in fatalities [3]. The vast majority of these accidents happened in built-up areas where only 25 per cent of total HGV mileage was covered.

Side-to-side collisions are the most common configuration of HGV-cyclist accidents. The Transportation Research Laboratory (TRL) investigated HGV-related accidents between 2006 and 2008 [3]. They reported that on average, HGVs cause 27 deaths and 72 serious injuries to cyclists each year in the UK, among which side-to-side collisions account for $43 \%$ of fatalities and $36 \%$ of serious injuries to cyclists. A second report from TRL which looked at data for a different period of time (2008 to 2011) also arrived at similar conclusion: the most common manoeuvre in $\mathrm{HGV}$-cyclist collisions was turning left: with a percentage being 31\% [4].

Two physical factors are implicated in side-to-side collisions between HGVs and cyclists. Firstly, large blind spots exist around HGVs, especially during cornering manoeuvres. Consequently the driver may not be able to see a cyclist in dangerous proximity. Secondly, HGVs often 'cut the corner' in urban streets, encroaching into the area where cyclists travel [5]. A simulation of a typical articulated HGV traversing a standard 90 degree left corner of radius $10 \mathrm{~m}$ showed that the vehicle can encroach into the inner boundary of the traffic lane by $1.6 \mathrm{~m}$ at maximum. In this same manoeuvre the side of the vehicle can approach the lane boundary at speeds of up to $1.4 \mathrm{~m} / \mathrm{s}$ [6]. Such a closing speed would leave a cyclist located in the danger zone unable to react sufficiently quickly to the vehicle's manoeuvre and an accident is very likely to occur.

There are a number of initiatives to address safety for cyclists and pedestrians for front/rear end collisions. For instance, Volvo's Cyclist Detection system harnesses a video camera to distinguish the types of objects in front of a car, and a radar unit to determine the distance to them [7]. Systems have been developed for protecting cyclists on the side of heavy vehicles, by 1), providing the driver with side-view cameras or wing mirrors for better visibility of blind spots 2), utilizing ultrasonic sensors on the side of the vehicle to detect cyclists who stray too close. To the authors' knowledge, these existing technologies give warning signals based on either detected distance or presence of an object in the detection range, without considering the motion information. Providing an alarm to the driver is not sufficient to reduce the chance of collisions, because the time available to prevent a collision is 
short, lorry drivers are heavily loaded with physical and cognitive tasks, and some might fail to respond promptly or correctly in many cases. To make matters worse, false alarms are distracting and annoying for the driver, and therefore may be ignored or shut down.

It is shown in [5] that some collisions between HGVs and cyclists could be prevented by suitable intervention in the motion of the vehicle. A braking manoeuvre, if introduced in time, could slow down or stop the HGV and let the cyclist escape. A Collision Avoidance System (CAS) is proposed in [6] with the objective of minimizing the likelihood of accidents between cyclists and HGVs, especially side-to-side collisions, by employing sensors to detect the relative motion of the cyclists and a collision avoidance strategy to automatically brake the vehicle when a potential collision is predicted at a fixed look-ahead time in the future.

A detailed simulation study for the proposed CAS was performed in [5], where two case studies were investigated. The first case study modelled articulated and rigid vehicles turning a fixed corner, alongside a cyclist. In each case, 560,000 different combinations of start positions and vehicle speeds were simulated in order to map out the outcomes of each encounter and determine the situations in which the CAS would be effective. It was concluded that the CAS could prevent $78-91 \%$ of the accidents involving articulated vehicle, and $64-88 \%$ of accidents involving the rigid truck. In the second case study, 19 real accidents were reconstructed from police case files using detailed information from witness and police statements, tachograph records, site photographs, etc. It was concluded that 18 out of the 19 fatalities could have been prevented by the proposed CAS. See details in [5]. On the basis in this analysis it was decided to build and test the system described in this paper.

Based on the research presented in [5] and [6], it was decided to build and test a prototype collision avoidance system (CA). This paper discusses testing of the prototype CAS in real time for verification of its effectiveness in preventing potential collisions between cyclists and HGVs. Section II summarises the proposed CAS in terms of cyclist detection and motion prediction. . Section III describes the testing setup for the prototype system while testing results are given in Section IV, followed by conclusions in Section V.

\section{COLlision AvoidAnCE}

\section{A. Overview}

There are three main stages in the proposed collision avoidance strategy: Detection, Prediction and Actuation, as shown

The first task in the Detection stage is to detect the cyclist and estimate its motion relative to the truck. During the same time, based on the current speed of the vehicle and its braking capability, a 'look-ahead' period, defined as 'Time-to-Avoidance' (TTA), is calculated. In the Prediction stage, information about the cyclist's motion relative to the HGV is sent to a processor to estimate the cyclist's position at time TTA in the future. In this estimation, it is assumed that the cyclist maintains its current acceleration (longitudinally and laterally), as well as its current yaw rate relative to the HGV. If a collision is predicted, an emergency (antilock brakes) stop is triggered automatically by the system during the Actuation stage.

This strategy only requires knowledge of the cyclist's motion relative to the HGV, without requiring HGV's position at any specific time. Using the relative motion means that accurate vehicle motion information, such as acceleration and position, is not required, eliminating the need for vehicle motion sensors and the associated computation.

\section{B. Cyclist Detection \\ 1) System formulation}

There are several possible sensing methods for detecting objects around a vehicle (summarized in [6]), including camera, RADAR, LIDAR and ultrasonic sensors. In this project, ultrasonic sensors were selected over the other detection methods because:

1) The characteristics of the detection geometry are well suited to cyclist detection. The lateral distance from the cyclist to the HGV is normally less than $2 \mathrm{~m}$; this corresponds to the detection range of a typical ultrasonic sensor

2) Ultrasonic sensors could provide distance measurements of acceptable accuracy.

3) The low cost of ultrasonic sensors make them commercially attractive.

The output from an off-the-shelf ultrasonic sensor is the distance between the target and the sensor. With the distance information alone, it is not possible to pinpoint the exact position of the target because the bearing angle from the sensor to the target is unknown. In order to construct the cyclist's trajectory relative to the truck, it is necessary to recover the bearing information.

A coordinate system was set to define the positions of the ultrasonic sensors on the truck as well as to describe the cyclist's position. The origin was set at the mid-point on the front edge of the vehicle, with $x$ axis along the longitudinal direction and $y$ axis pointing to the left.

A reference line (UA) was defined for each sensor, pointing along the outwards normal to the side of the vehicle (as shown in Fig. 2). The position vector from the sensor to the target is UB. The angle from the reference line to the position vector is defined 
as the target bearing $\theta$. Clockwise rotation of $\theta$ is defined as positive.

Given the lateral and longitudinal positions for the ultrasonic sensor $\left(P_{s x}, P_{s y}\right)$ in this coordinate system and the detected distance $d$ (the length of the UB line defined in (Fig. 2) the cyclist position $\left(P_{c x}, P_{c y}\right)$ can be expressed by the following equations:

$$
\begin{aligned}
& P_{c x}=P_{s x}+d \cdot \sin \theta \\
& P_{c y}=P_{s y}+d \cdot \cos \theta
\end{aligned}
$$

It is impossible to solve (1) and (2) independently, as the number of equations is smaller than the number of unknowns, rendering it an undetermined system mathematically.

2) Quadratic Programming

Given a series of detected distances $\left(d_{1}, d_{2}, d_{3}, \ldots, d_{n}\right)$ at sampling times $\left(t_{1}, t_{2}, \ldots, t_{n}\right)$, we need to find the corresponding bearings $\left(\theta_{1}, \theta_{2, \ldots}, \theta_{\mathrm{n}}\right)$ so that the cyclist positions $\left(P_{c x_{1}}, P_{c x_{2}}, \ldots, P_{c x_{n}}\right)$ and $\left(P_{c y_{1}}, P_{c y_{2}}, \ldots, P_{c y_{n}}\right)$ can be determined. Based on $(1)$ and (2), the longitudinal acceleration of the cyclist can be expressed as a function of the bearings, with detected distances and sensor positions being the known parameters. Assuming constant mean longitudinal acceleration for the cyclist, it is possible to find the best set of $\theta_{i}(i=1, \ldots, n)$ that would result in a smooth trajectory history for the cyclist that minimizes the variation in longitudinal acceleration.

$$
J=\sum_{k=3}^{n}\left(A_{c x, k}-\bar{A}_{c x}\right)^{2}
$$

where, $\bar{A}_{c x}$ is the mean longitudinal acceleration of the cyclist during the period to inspect. This can be achieved by formulating a classic quadratic programming $(\mathrm{QP})$ problem.

$$
J=\frac{1}{2} \Theta^{T} Q \Theta+L \Theta
$$

with equality constraints:

$$
A_{e q} \boldsymbol{\Theta}=B_{e q}
$$

and inequality constraints:

$$
A_{\text {in }} \Theta \leq C_{\text {in }}
$$

Here $\Theta$ is the vector of bearing angles, $Q$ is the quadratic matrix and $L$ is the linear matrix. $A_{e q}, B_{e q}, A_{\text {in }}$ and $C_{i n}$ in (5) and (6) are matrices that determines the relation between elements in $\boldsymbol{\Theta}$.

The equality constraints (5) are generated using triangulation, in cases where two neighbouring sensors have overlapped detection ranges. The inequality constraints (6) occur because (i) each value of $\theta_{\mathrm{i}}$ must be limited by upper and lower sensing limits, based on the range of field of the sensor; (ii) there are some simple consistency requirements for the order of the detections, based on the relative motion.

In practice, there are some inaccurate detections (ultrasonic pings are not always reflected from the same point of the cyclist) and signal dropouts, it is not possible to obtain a smooth trajectory for the cyclist purely based on quadratic programming and triangulation. A Kalman filter is used to smooth the results derived from quadratic programming based on a kinematic model of the cyclist motion. Fig. 3 summarized the steps taken to estimate the cyclist's position relative to the truck in real time. A detailed description of the estimation of the cyclist motion based on ultrasonic sensors is presented in [6] and [8].

\section{Time-to-Avoidance}

In this study the Time-to-Avoidance (TTA) is an estimate of the time from the detection of a potential collision to the moment when the vehicle is brought to full stop under maximum braking torque.

$$
T T A=t_{s}+\frac{V}{\mu g}
$$

Here: $t_{s}$ represents the system delay time, including the times for sensing, processing and brake actuation. $V$ is the vehicle speed, $\mu$ is the road-tyre friction coefficient and $g$ is the gravitational acceleration. Within the TTA, the HGV can fully stop and a collision can just be avoided. Even if a cyclist is still in motion at the point of impact, the severity of the collision could be greatly reduced.

\section{Motion Prediction}

The acceleration $A_{c}$ and velocity $V_{c}$ of the cyclist cannot be measured directly; however, they can be derived from the cyclist's 
position using numerical differentiation. Five sample points are used to evaluate the acceleration and the velocity terms. The future positions of the cyclist can be found using Euler integration by running the following set of equations in loop from time $t$ to time $t+T T A$.

$$
\begin{aligned}
& V_{c}(t+d t)=V_{\mathrm{c}}(t)+A_{c} d t \\
& P_{c}(t+d t)=P_{c}(t)+V_{c} d t
\end{aligned}
$$

\section{E. Collision Assessment and Intervention}

After the motion of a cyclist relative to the HGV has been predicted, it is necessary to define a criterion to judge whether a potential collision is imminent. When the envelope of the predicted cyclist position overlaps the HGV's envelope, a collision signal is issued.

A 'threshold distance' is introduced to tune the decision about when to intervene with the brakes. The HGV brakes are activated when the predicted cyclist's distance to the side of the vehicle is less than the threshold distance at look-ahead time TTA. A larger threshold distance will cause the vehicle to decelerate sooner to avoid an accident, but will also cause more unnecessary 'false positive' interventions. The threshold distance was determined from the results of a set of simulations. A value of $0.15 \mathrm{~m}$ was found to be a good compromise for tests in which the vehicle speed was less than $12 \mathrm{~km} / \mathrm{h}$.

\section{TESTING SETUP FOR THE PROTOTYPE SYSTEM}

\section{A. Overview}

A program of full-scale vehicle tests was conducted between April and June 2014. The testing aimed to prove that the suggested CAS can:

1) detect and estimate the motion of a cyclist in real time;

2) predict the likelihood of a collision between cyclist and truck;

3) activate the braking system of the truck when necessary to prevent collisions

There was no standard testing procedure available for testing a collision avoidance system in the literature, either for sidecollisions or HGV-cyclist interactions. Therefore, a new testing methodology was developed.

The test setup involves a real truck and a dummy cyclist (simplified as 'dummy' in the following context), propelled by a motorized belt. Fig. 4 shows a schematic of the testing setup as well as the key equipment used for the vehicle testing. All equipment on the truck is termed the 'truck-based system' and the rest is called the 'road-side system'. Each piece of testing equipment will be discussed in the following sections.

The manoeuvre chosen for testing was a left-turning truck crossing the path of the cyclist, which was moving in a straight line, as shown by the dash dot arrow lines in Fig. 4. This was found to be the most common accident configuration in an analysis of real accidents in the UK [5]. The dummy was set up to move at a designated speed and following a straight path. The truck followed a pre-defined left-turning path at a given speed. The truck's path was designed to intersect with the dummy's path. Multiple ultrasonic sensors were fitted on the left side of the truck for cyclist detection. The computer controller was placed in the cab for real time control. The truck brakes were activated when a potential collision is predicted. Further details are provided below.

\section{B. $\quad x P C$ Model and Data Exchange in Real Time}

A real time CAS controller was developed using xPC Target for real time control from Mathworks [9]. A single Simulink model was developed for the vehicle testing. This Simulink model has a number of blocks that deal with different functionalities of the CAS:

1) Ultrasonic sensor message input;

2) Cyclist position estimation;

3) Cyclist position prediction;

4) Collision assessment;

5) Actuation signals output.

An analogue to CAN device, called PCAN-MicroMod Analog (PCAN) from PEAK System®, was used to convert analogue signals from ultrasonic sensors into CAN messages. The PCAN has eight analogue inputs and four analogue outputs, working at high-speed CAN (ISO 11898-2) with the default data transmission rate as 500k Baud. The inputs can measure bipolar range, from -10 to $+10 \mathrm{~V}$, with a 16-bit resolution.

In the XPC model, the multitasking mode was used to enable to different blocks in the model to run at different sampling rates. The basic sampling rate is clocked at $100 \mathrm{~Hz}$ (i.e., $0.01 \mathrm{~s}$ sample time) for the entire model and all the CAN-related devices (RT3000 Inertial and GPS Navigation System from OxTS® and PCAN) ran at this sampling rate. A slower sampling rate was needed for the cyclist position estimation and prediction blocks which take longer than $0.01 \mathrm{~s}$ to complete. As the refresh rate of the ultrasonic sensor output is $7.5 \mathrm{~Hz}$, it takes approximately $0.133 \mathrm{~s}$ to receive a new detection, which is sufficient for processing 
the estimation and prediction blocks. The xPC requires that the slower sample time must be an integer multiple of the faster sample time. Consequently, $0.13 \mathrm{~s}$ was chosen as the slow sample time instead of $0.133 \mathrm{~s}$. The $3 \mathrm{~ms}$ ' mismatch with the ultrasonic sensor's output rate could result in 3 missed sensor readings during a 20 s period of time, which was acceptable in this application.

\section{C. $\quad$ Testing Set-up}

The dummy cyclist consisted of a half body mannequin was mounted onto a thin wooden base via a tripod consisting of three aluminium alloy tubes (Fig. 5). A Soft Pedestrian Target (SPT) system from Anthony Best Dynamics Ltd (ABD) was used to pull the dummy along a $50 \mathrm{~m}$ straight path at steady speeds up to $4 \mathrm{~m} / \mathrm{s}$ [10].

The SPT was propelled by a continuous flat belt, connected to the dummy's wooden base via two quick release mechanisms. In the event of the truck wheel running over the belt, the belt was instantly released from the wooden base and the motor went open loop while the whole system was stopped.

The speed profile for the dummy was defined for each run of the test. A typical profile used for the testing consists of a short acceleration stage to get to the target speed, a constant speed stage and a deceleration stage to stop the dummy.

The SPT was triggered by the signal from the front wheel of the truck passing over a pressure mat (Fig. 4).

A Scania tipper truck provided by Laing O'Rourke was used as the testing vehicle for the project. Twelve ultrasonic sensors were used to cover the entire left side of the truck. The ultrasonic sensors, as shown in Error! Reference source not found., were placed at the height of $1.4 \mathrm{~m}$ above the ground, so that the sensors faced the dummy's mid torso.

For the braking actuation, a pedal robot ('sBrake' from Anthony Best Dynamics) [11] was mounted in the cab. When commanded, it applied a force to the brake pedal to stop the truck. The sBrake was attached onto the floor inside driver's foot well. . In a future development, the braking command will be sent directly to the Braking ECU, eliminating the need for the sBrake.

The RT3000 was used for measuring the vehicle motion. The outputs from the RT3000 are derived from measurements of its accelerometers and gyros, with a Kalman filter used to update the inertial measurements using GPS. The measurements were output over CAN bus. On the road side, a RT-base GPS base station was used to provide Differential GPS to improve position accuracy of the RT3000. The model used has a position accuracy of $20 \mathrm{~cm}$.

During the prototype testing, TTA was not calculated based on Equation (7), due to the lack of the estimation capability of road-tyre friction coefficient. As the RT3000 records the speed profile of the truck accurately, TTA can be measured directly. For each vehicle speed used in testing, the stopping time was measured using the RT3000 beforehand. An extra 0.2s was added to the measured stopping times to give slightly more conservative behaviour. The inclusion of real time estimation of TTA will be carried out in a subsequent project. There were two separate data collection systems. The position of the dummy along the belt relative to the motor was recorded by the SPT software on a road-side laptop. The dummy positions estimated by the CAS and other vehicle data including its absolute position were stored in a laptop on the truck. There was no direct link between two sets of data and each set of data had its own time stamps. To indicate the common start point for both sets of data and to verify the estimated positions of the dummy against its true positions, a communal time stamp was inserted into the two sets of data. A wireless link was therefore included between the road-side instrumentation system and the vehicle-based system.

\section{TESTING RESUlts}

\section{A. Stationary Truck Testing}

The first objective of the testing was to check whether the CAS could detect a moving target consistently and estimate the position correctly. The second was to determine the range of relative speeds between the dummy and the vehicle for which reliable detections could be obtained by the CAS. The prediction of the cyclist's future position and the collision assessment were not verified in the stationary truck testing stage.

Various relative speeds between the truck and the dummy were used, with the maximum speed differential set as $5 \mathrm{~km} / \mathrm{h}$. This speed limit is determined by the sensing performance of the ultrasonic sensors. If the relative speed is greater than $5 \mathrm{~km} / \mathrm{h}$, the dummy cyclist fails to be detected consistently due to the low refresh rate of the sensors. It is hoped to improve this maximum speed in a future system through use of higher performance ultrasonic sensors. During each test run, the speed combination and the time delay were set in a way that the dummy was in the detection range of the sensors for a longest possible period time. 
An example is provided in

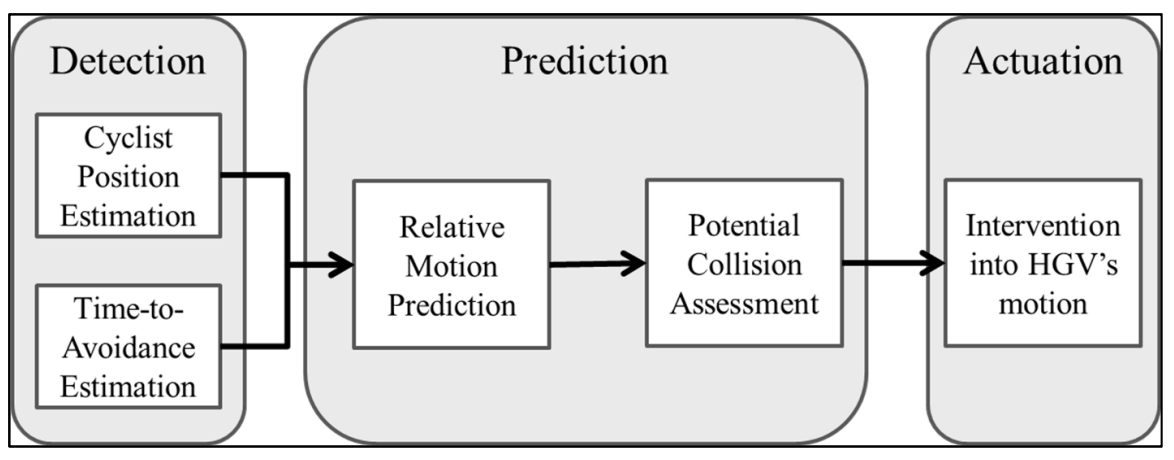

Fig. 1. Control Structure of the Collision Avoidance System

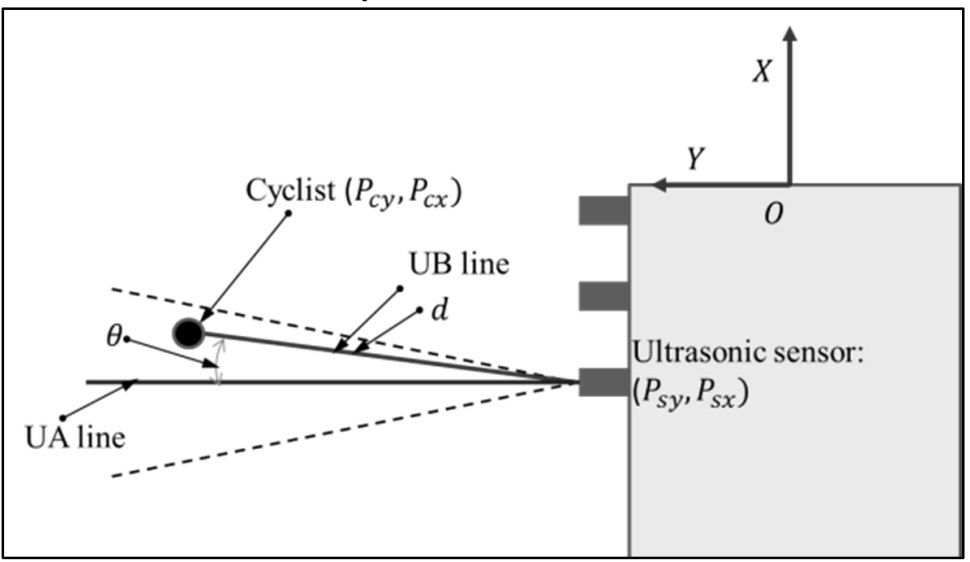

Fig. 2. Illustration of the cyclist detection

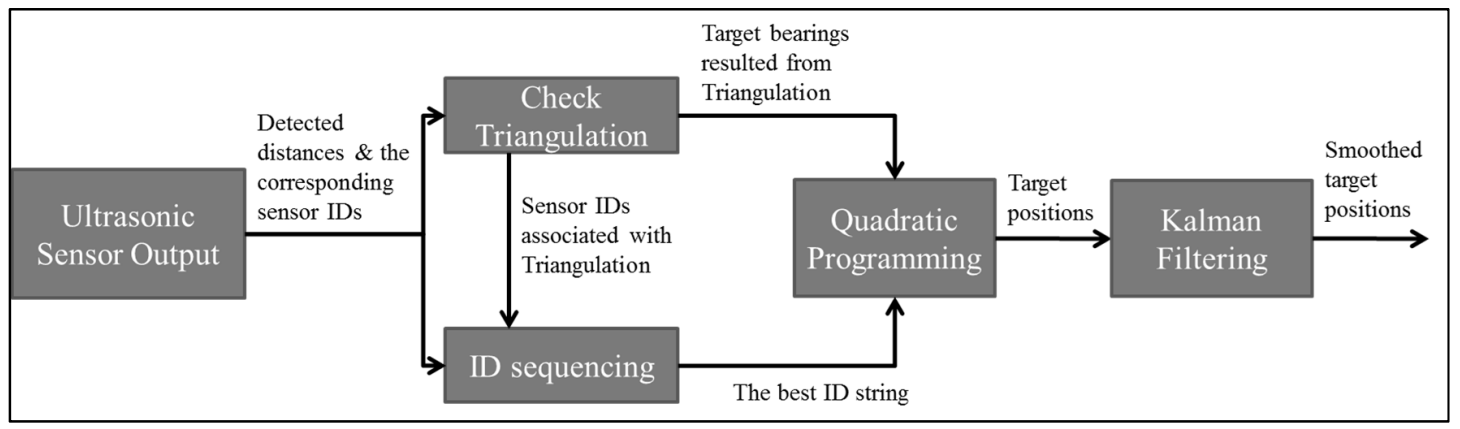

Fig. 3. A flowchart for position estimation for cyclist in real time

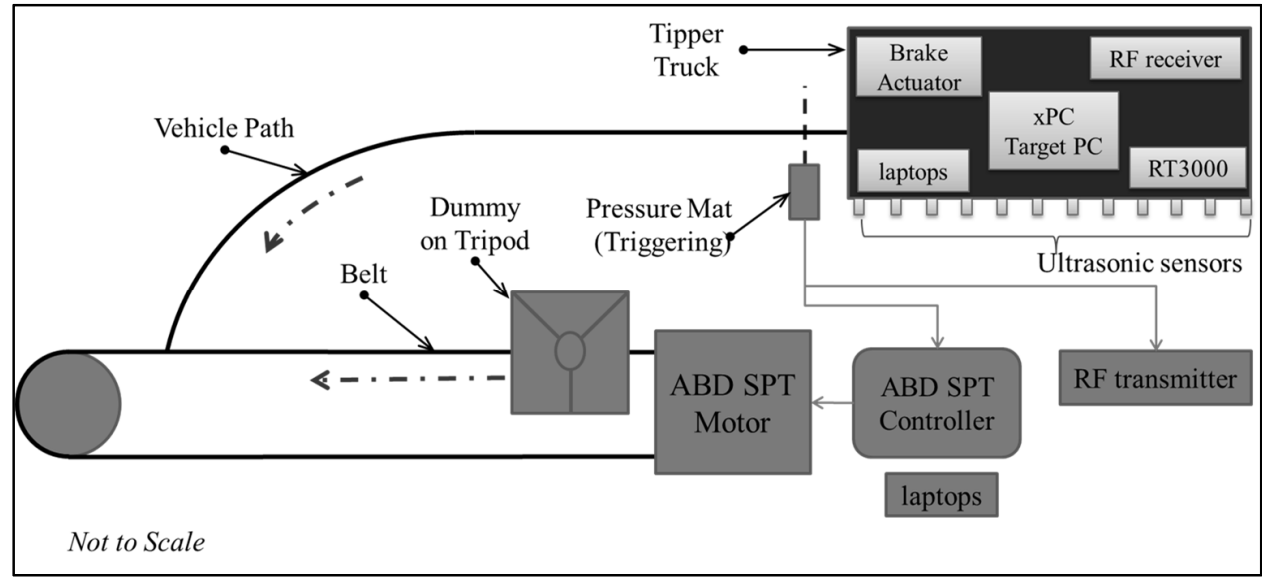

Fig. 4. The schematic for testing the collision avoidance system 


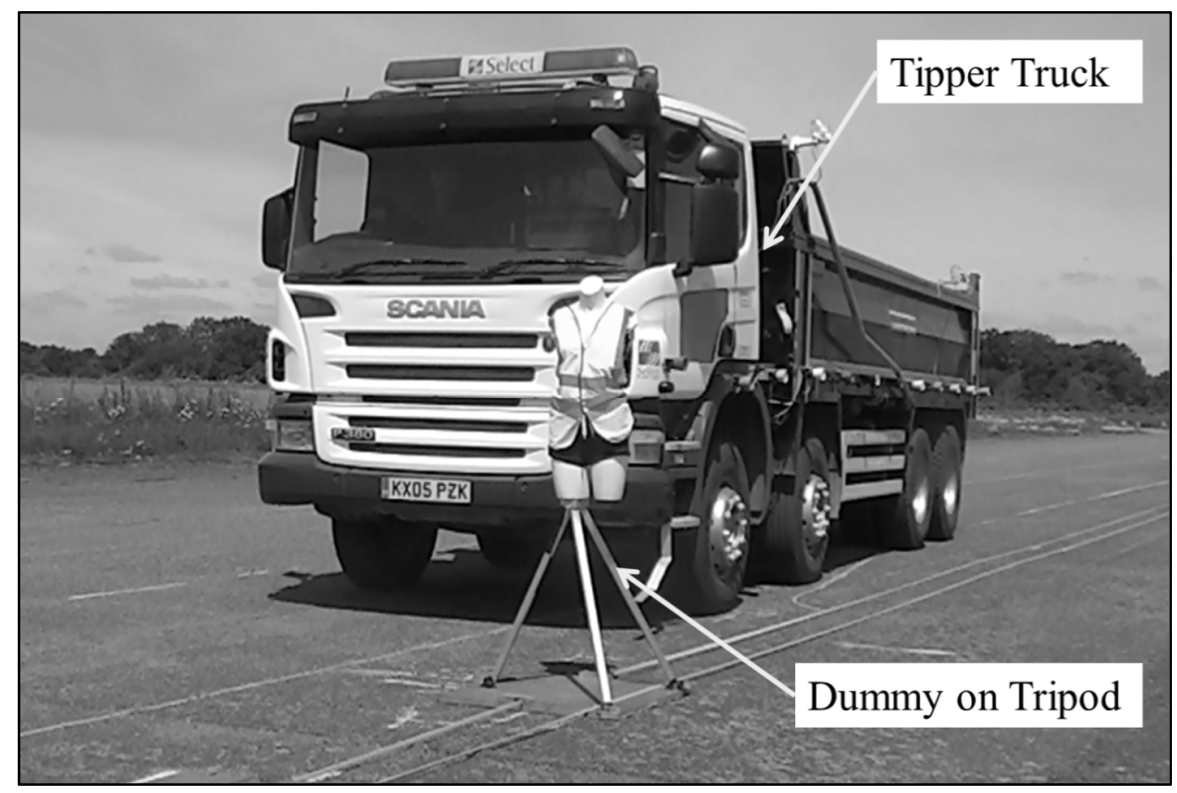

Fig. 5. Testing setup showing the truck \& the dummy cyclist

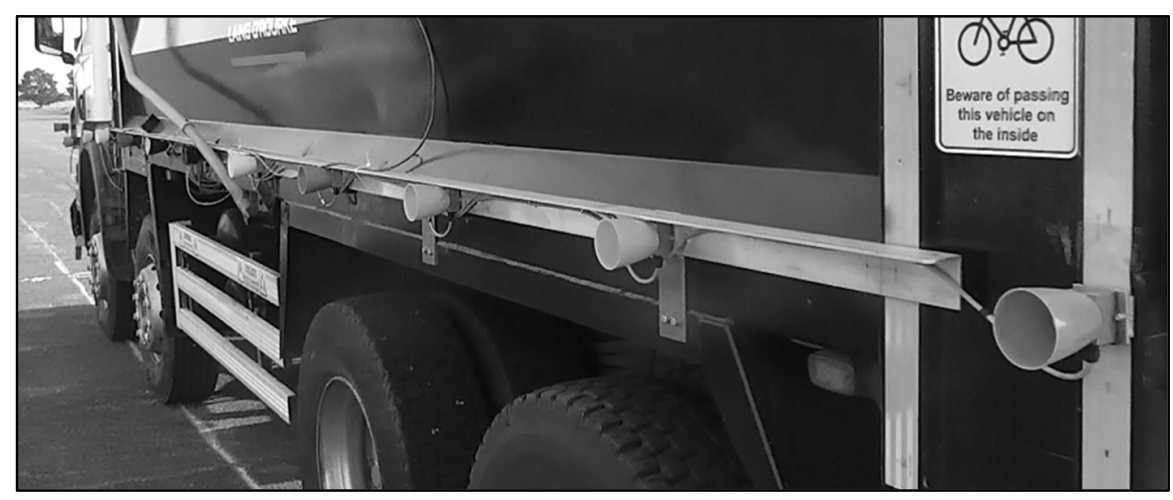

Fig. 6. Ultrasonic sensors mounted along the side of the test vehicle

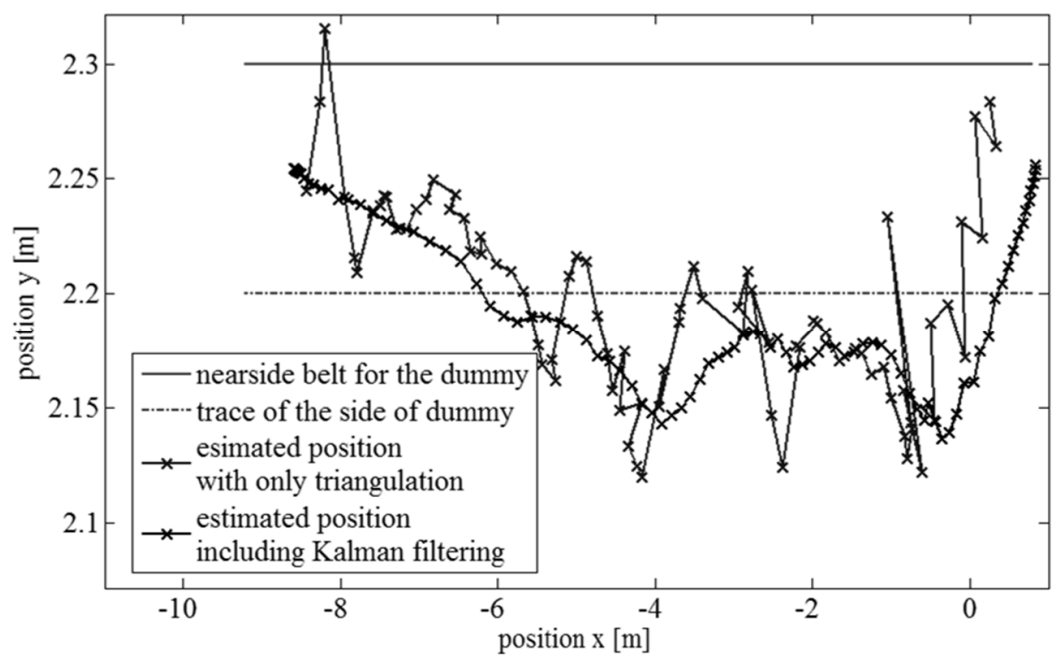

Fig. 7, where dummy speed was $3 \mathrm{~km} / \mathrm{h}$. It is noticeable that the Kalman filter (black crosses) significantly improves the estimation errors of the QP and gives a much smoother trajectory. Another observation from the figure is that the maximum error in lateral position was approx. $5 \mathrm{~cm}$. The standard deviation of the estimation errors and maximum detection errors for 5 different relative speeds are provided in Error! Reference source not found.. These results proved that the CAS could track the dummy to a level of accuracy that is acceptable (5-10cm maximum errors). 
It was also important to check if the similar performance could be achieved when the cyclist trajectory was angled towards the truck. Such a testing scenario was termed 'diagonal testing'. One configuration of such diagonal testing is shown in Fig. 8. The error characteristics are summarized in Error! Reference source not found.. It is concluded that whether the configuration is diagonal or parallel, the cyclist position estimator using the combination of QP and Kalman filter work to a similar level of accuracy.

From the discussion above, the following conclusions can be drawn:

1) The estimated trajectories in all the figures followed the dummy's real trajectory in a reasonable way.

2) The maximum estimation errors were within $10 \mathrm{~cm}$.

3) The $1 \mathrm{~km} / \mathrm{h}$ case (i.e. small relative speed) was the worst in terms of tracking oscillations and estimation errors.

4) The estimated trajectory for the $3 \mathrm{~km} / \mathrm{h}$ case offered the best results compared with other speed cases.

5) When the relative speeds were higher, the oscillations reduced. However, the ultrasonic sensors performed worse because less raw detection was made.

\section{B. Moving Truck Testing}

It was of interest to check if similar results could be obtained when the truck is in motion. A line parallel to the nearside belt of the SPT system was drawn on the ground, which served as the left boundary of the truck path. The distance between the nearside belt and the line was set to $1.2 \mathrm{~m}$. An L-shape pointer was placed near the front left corner of the truck. It could be observed by the truck driver from the top mirror which pointed to the ground. The truck driver maintained a straight line by referring to the position of the L-shape pointer relative to the line on the ground.

A typical example of the dummy overtaking the truck in parallel is provided in Fig. 9. The dummy was set with a speed of $6 \mathrm{~km} / \mathrm{h}$ and the truck was maintained at a speed around $3 \mathrm{~km} / \mathrm{h}$. It can be seen that the estimated position of the dummy (line with crosses) was very close to dummy's true position (light dash line), and the error level is less than $10 \mathrm{~cm}$.

To test the scenario that a left turning truck closes in onto the dummy's path, instead of having the truck follow a naturallycurved left turn, it was much simpler to let the vehicle follow a straight line parallel to the dummy and then turn onto a diagonal path that intersected the path of the dummy. Similar to the parallel testing cases, different speeds were assigned to both the vehicle and the dummy, keeping the speed difference less than $5 \mathrm{~km} / \mathrm{h}$.

A typical example is illustrated in

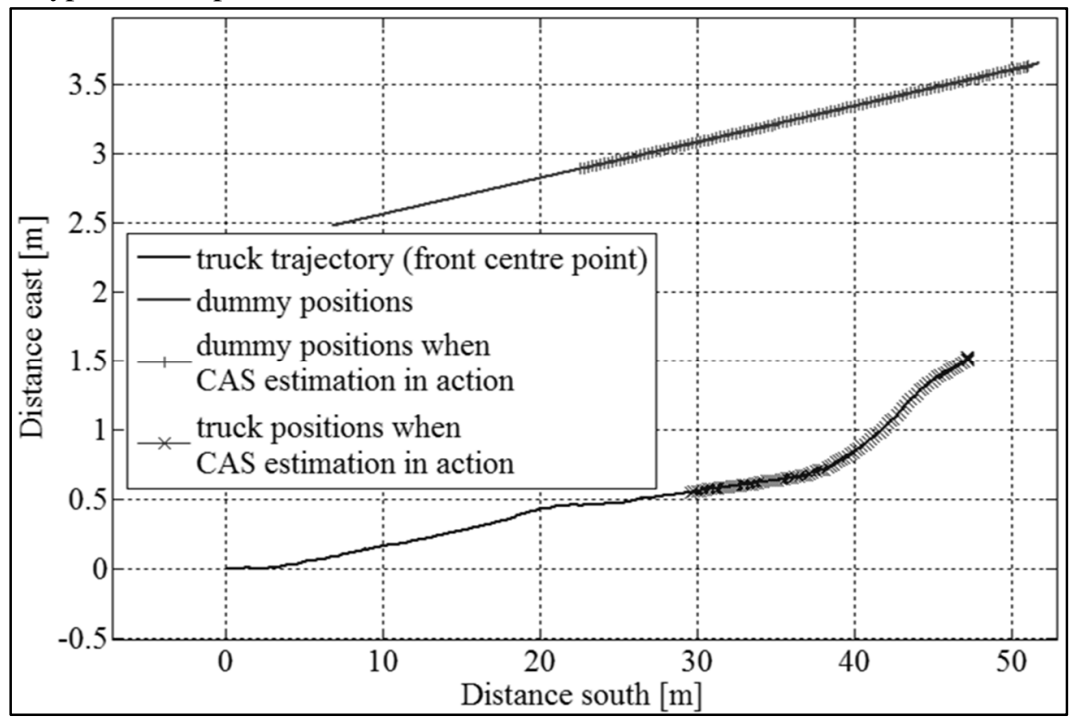

Fig. 10 to Fig. 12. In this example, the speed for the dummy was $7 \mathrm{~km} / \mathrm{h}$ and the truck speed was $5 \mathrm{~km} / \mathrm{h}$. 


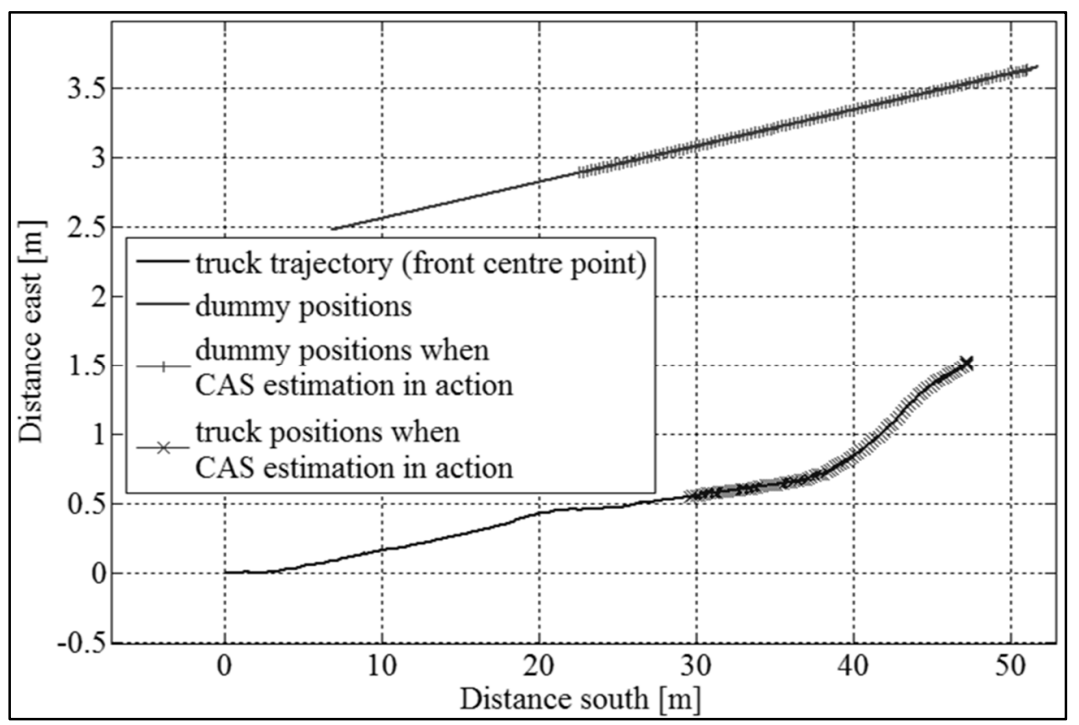

Fig. 10 shows the truck's trajectory and the dummy's track in the global coordinate system. The path is almost due South. It can be seen that the truck narrowed the gap between it and the dummy at approximately $37 \mathrm{~m}$ through the $50 \mathrm{~m}$ long test.

The CAS predicted the future positions of the dummy while estimating its current position. It can be seen in Fig. 11 that the estimated positions were off the target line by a few centimetres on the straight-line section. The CAS captured the dummy's motion well for the majority of the left-turning path. Fig. 11 also compares the predicted positions against the reference line, with the estimated positions plotted in the same figure as well. It can be seen that the prediction worked well, although a level of mismatch between the trajectories can be observed. This level of inaccuracy was still within $10 \mathrm{~cm}$.

The threshold distance line was plotted as a dotted line in Fig. 11. This line was shifted away from the sensor positions by $566 \mathrm{~mm}$ (half of the diagonal of the dummy's base) with its lateral coordinates being $1.86 \mathrm{~m}$ from the centre line of the truck. This line was designated the 'collision assessment line'. The brakes were actuated immediately the predicted position crossed the collision assessment line (i.e. at $\mathrm{x}=-1.3 \mathrm{~m}$ in Fig. 11).

The speed profiles of both the dummy and the truck are plotted Fig. 12. The lateral positions of the dummy (both current and future positions) are also plotted against the same time scale. It can be seen that the future lateral position crossed the $1.86 \mathrm{~m}$ line at $32.9 \mathrm{~s}$. The vehicle started to decelerate rapidly shortly after this time. The truck speed reached zero at $34 \mathrm{~s}$. The dummy was about $0.5 \mathrm{~m}$ away from the truck laterally at that time.

At the end of the entire testing period, the CAS was switched off for a run in which the dummy was deliberately hit by the moving lorry. This run served as the control for a CAS-enabled run. The truck speed was $8 \mathrm{~km} / \mathrm{h}$ and the dummy $10 \mathrm{~km} / \mathrm{h}$.

The speed profile for both the truck and the dummy in this control test are shown in Fig. 13. It can be seen that when the dummy position crossed the collision assessment line there was no reduction in truck speed. Both the dummy and the

truck proceeded without slowing down until they reached a point (approx. 21s) when the lateral distance was $1.3 \mathrm{~m}$, off the side of the truck. The dummy hit the truck and the speed of the dummy oscillated wildly.

If the CAS was enabled, the braking command would have begun stopping the lorry immediately after the predicted position of the dummy (light solid line in Fig. 14) crossed the collision assessment line.

The effectiveness of the CAS for a matrix of left-turning truck cases is summarised in Table 3. A successful prevention of a potential accident was defined as the truck being stopped by the CAS without intervention by the driver. This was marked as a tick $(\checkmark)$. If the test run was terminated by any human inputs, the result was treated as a failed test as indicated by a cross sign ( $\mathbf{x}$ ) in the table. The speed combinations that were untested were left blank.

For each test case three repeats were performed, however, these repeats were not identical because both the truck position and speed varied slightly under manual control. Failed runs are denoted by the number in the brackets in the table. Of all the test runs, only 4 failed detections occurred. These were due to some of the sensors failing to detect the dummy for a period of time; therefore the system failed to respond correctly.

\section{CONCLUSIONS}

1) An overview of the collision avoidance system that focuses on preventing collisions between the HGVs and the cyclists was presented. A testing methodology was developed for testing the proposed side collision avoidance system. It consisted of a motorized dummy cyclist and a moving truck.

2) The ultrasonic sensors used in this project provided relatively reliable detections when the speed differential between the truck and the dummy cyclist was less than $5 \mathrm{~km} / \mathrm{h}$.

3) Preliminary testing with the truck stationary and a moving cyclist proved that the quadratic programming can be used in combination with a Kalman filter to determine the 
cyclist position within $10 \mathrm{~cm}$ for a $5 \mathrm{~km} / \mathrm{h}$ speed differential.

4) The predicted position of the cyclist, under the constant acceleration assumption, was within reasonable accuracy.

5) The moving truck testing showed that vehicle-based detection and estimation worked well for constant speed tests. The testing provided similar results to the stationary testing, which further demonstrated that the CAS could work effectively for a range of relative motions. Potential collisions were prevented successfully by this prototype CAS system, and the performance was reliable within a $5 \mathrm{~km} / \mathrm{h}$ speed difference between the dummy and the truck. With a higher relative speed, the ultrasonic sensors failed to provide consistent detections and the system's performance began to degrade.

\section{ACKNOWLEDGEMENTS}

The authors thank the support of the Cambridge Vehicle Dynamics Consortium, whose member at the time of writing are: Anthony Best Dynamics, Camcon, Cambridge University, Denby Transport, Firestone Goodyear, Haldex, Laing O'Rourke, MIRA, SDC Trailers, SIMPACK, Tridec, Tinsley Bridge, Wincanton and Volvo Trucks. Special thanks go to Anthony Best Dynamics and Laing O'Rourke for proving essential testing equipment. Thanks also go to Dr Richard Roebuck, Dr Leon Henderson and Ms Amy Rimmer for their assistance with the testing. The authors also would like to thanks China Scholarship Council and Cambridge Trusts for their contribution to the research.

VII. TABLES

\begin{tabular}{|c|c|c|}
\hline $\begin{array}{c}\text { Dummy speed } \\
\text { relative to the } \\
\text { stationary truck (in } \\
\mathrm{km} / \mathrm{h} \text { ) }\end{array}$ & $\begin{array}{c}\text { Mean } \\
\text { lateral errors } \\
\text { (in cm) }\end{array}$ & $\begin{array}{c}\text { Standard } \\
\text { deviation of } \\
\text { lateral errors (in } \\
\mathrm{cm} \text { ) }\end{array}$ \\
\hline 1 & -1.3 & 4.0 \\
\hline 2 & -0.7 & 3.6 \\
\hline 3 & 1.0 & 4.1 \\
\hline 4 & 0 & 4.8 \\
\hline 5 & 2.9 & 3.4 \\
\hline
\end{tabular}

\begin{tabular}{|c|c|c|}
\hline $\mathrm{km} / \mathrm{h})$ & & $\mathrm{cm})$ \\
\hline 1 & -0.1 & 4.3 \\
\hline 2 & 0.2 & 2.3 \\
\hline 3 & 1.1 & 2.8 \\
\hline 4 & 1.8 & 3.4 \\
\hline 5 & -0.6 & 1.0 \\
\hline
\end{tabular}

Table 2 Position estimation accuracy for cyclist moving diagonally w.r.t. a stationary truck

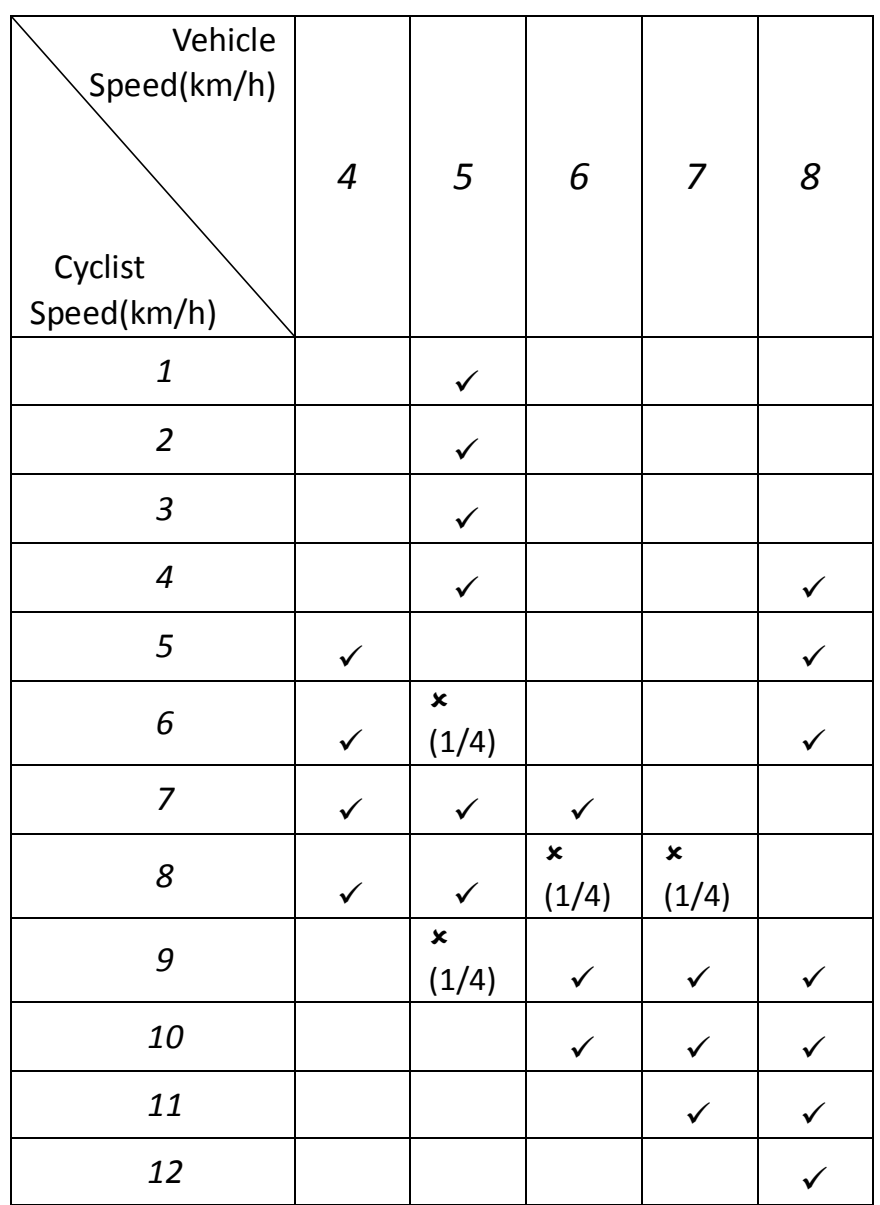

Table 3. Test results for a left-turning truck with a straight line dummy: $(1 / 4)$ means a failure on 1 out of 4 repeated tests

Table 1 Position estimation accuracy for cyclist moving in parallel with a stationary truck

\begin{tabular}{|c|c|c|}
\hline $\begin{array}{c}\text { Dummy speed } \\
\text { relative to the } \\
\text { stationary truck (in }\end{array}$ & $\begin{array}{c}\text { Mean } \\
\text { lateral errors } \\
\text { (in cm) }\end{array}$ & $\begin{array}{c}\text { Standard } \\
\text { deviation of } \\
\text { lateral errors (in }\end{array}$ \\
\hline
\end{tabular}

VIII. FIGURES 


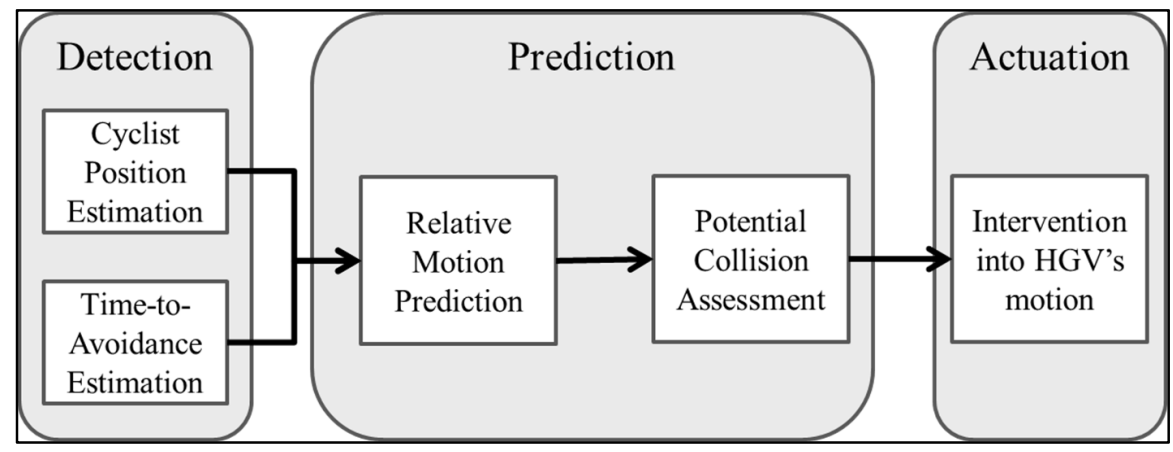

Fig. 1. Control Structure of the Collision Avoidance System

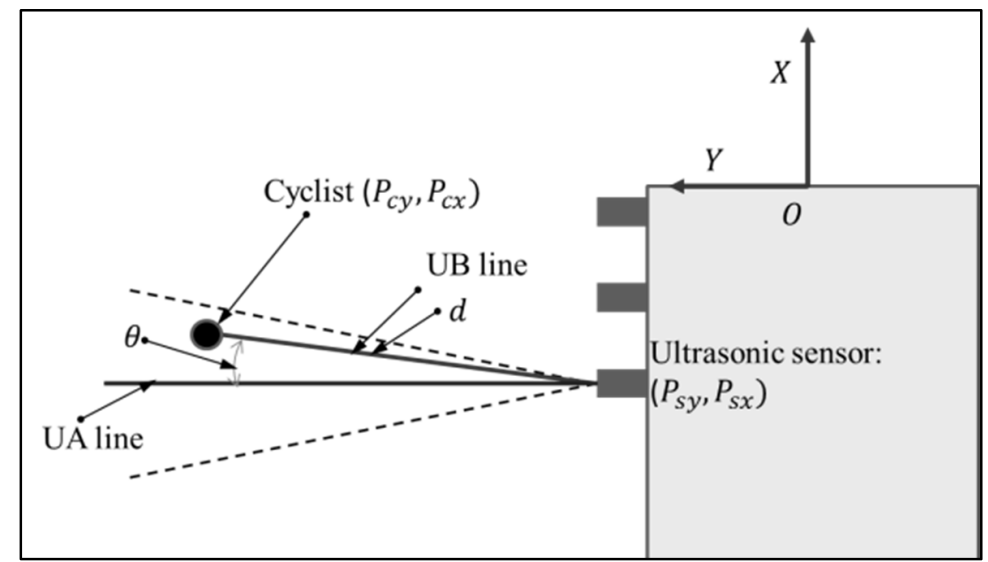

Fig. 2. Illustration of the cyclist detection

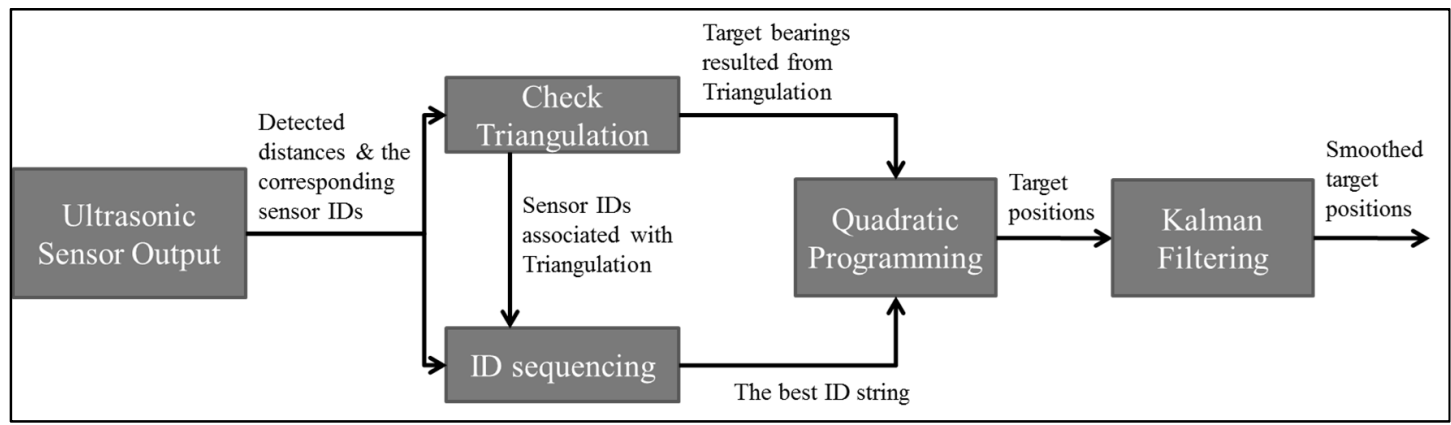

Fig. 3. A flowchart for position estimation for cyclist in real time

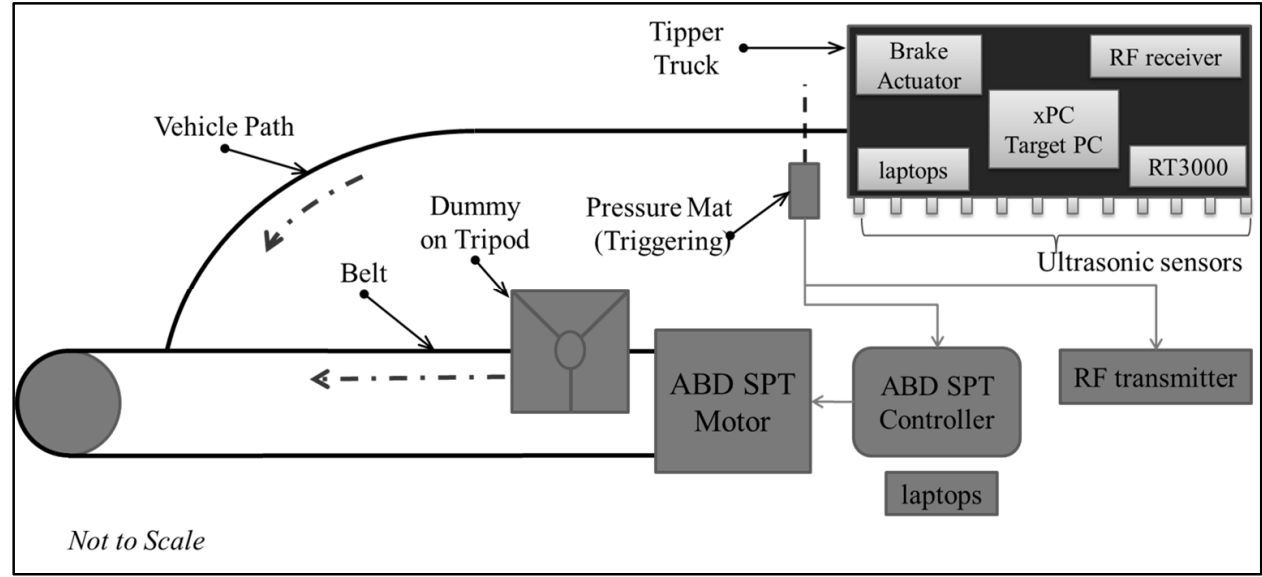

Fig. 4. The schematic for testing the collision avoidance system 


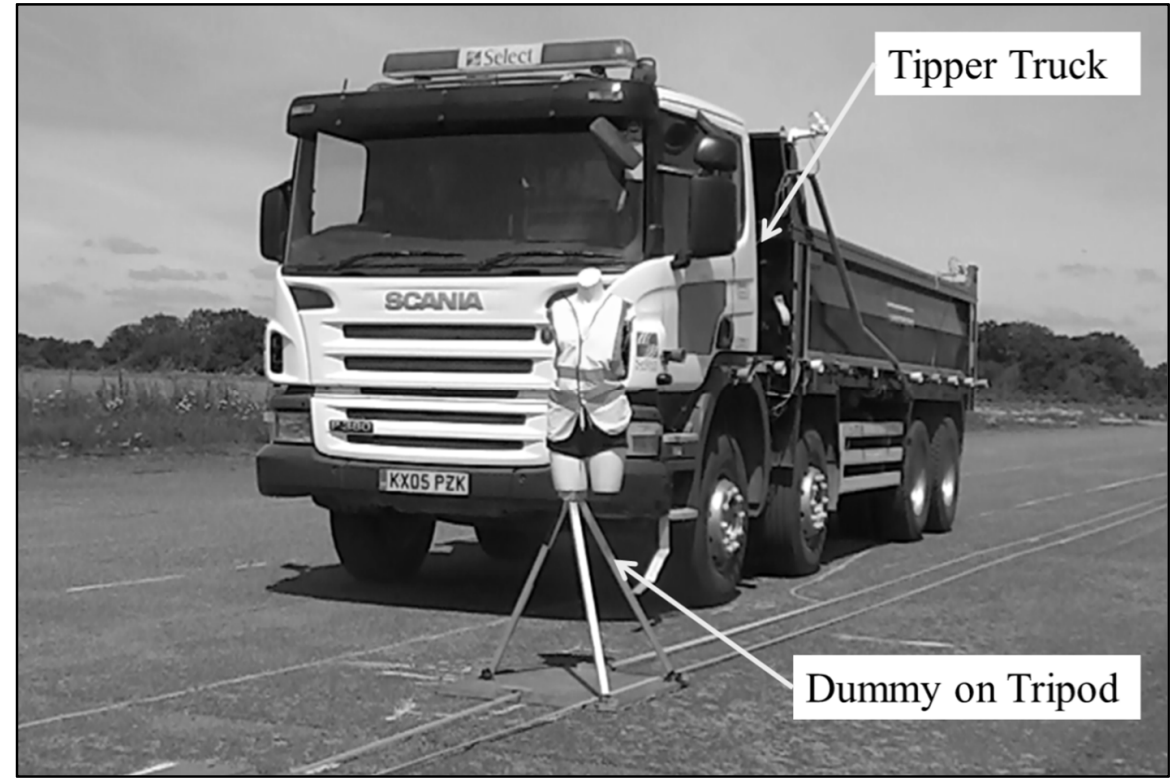

Fig. 5. Testing setup showing the truck \& the dummy cyclist

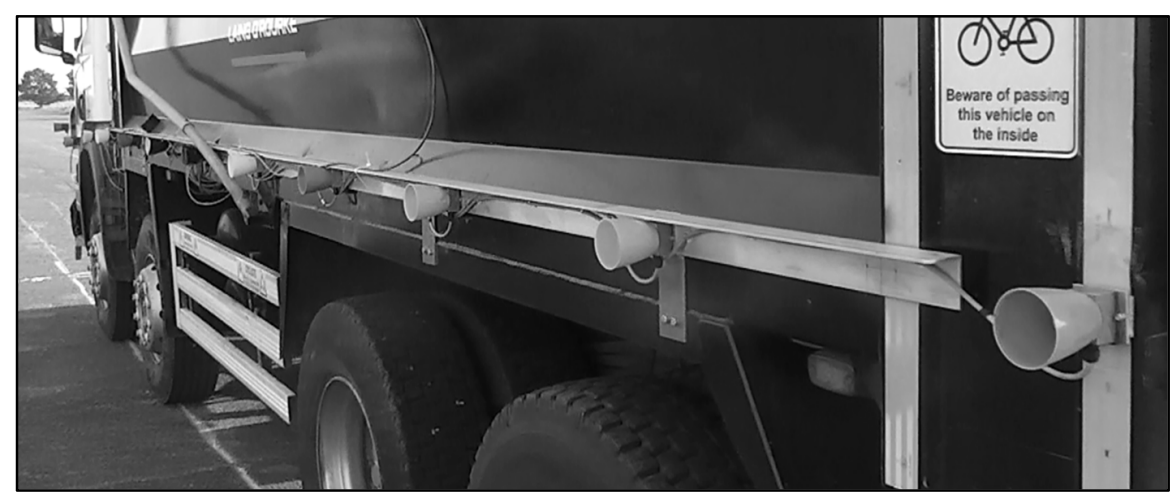

Fig. 6. Ultrasonic sensors mounted along the side of the test vehicle

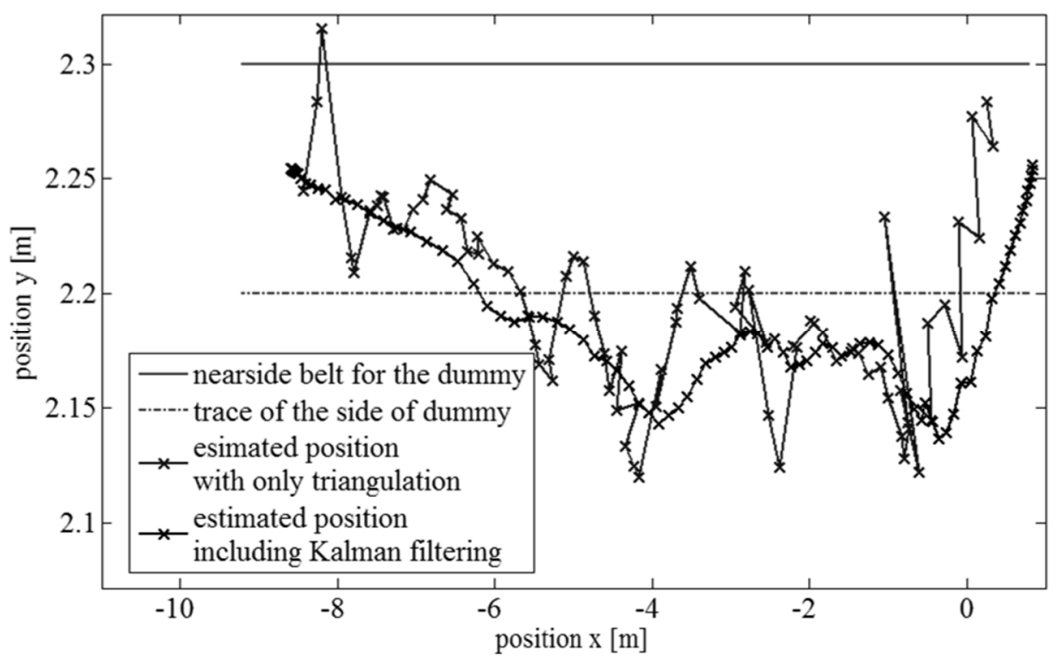

Fig. 7. Testing result of a moving dummy at $3 \mathrm{~km} / \mathrm{h}$ in parallel with a stationary truck 


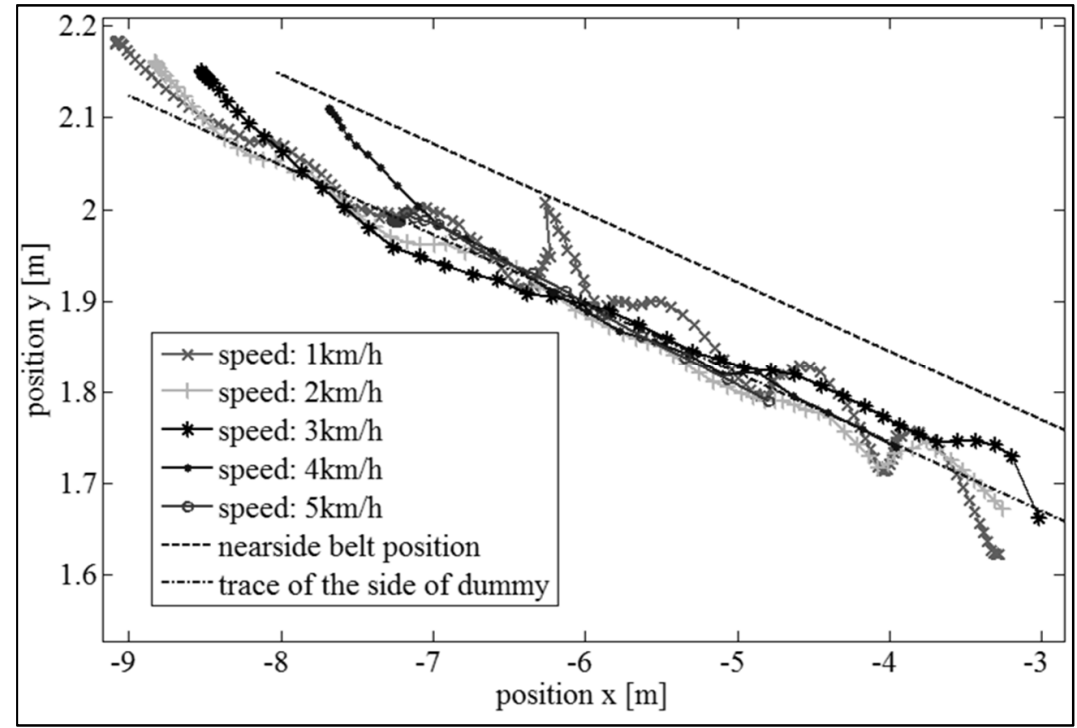

Fig. 8. Estimated position comparisons for different speeds for the dummy travelling diagonally

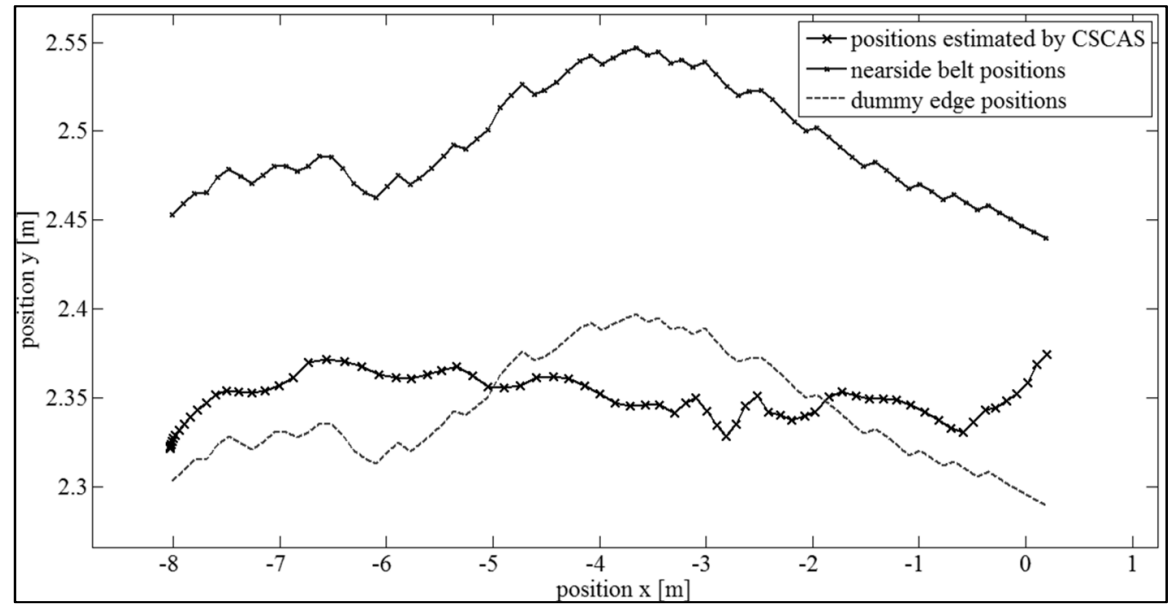

Fig. 9. Parallel testing result with the dummy $(6 \mathrm{~km} / \mathrm{h})$ overtakes the truck $(3 \mathrm{~km} / \mathrm{h})$

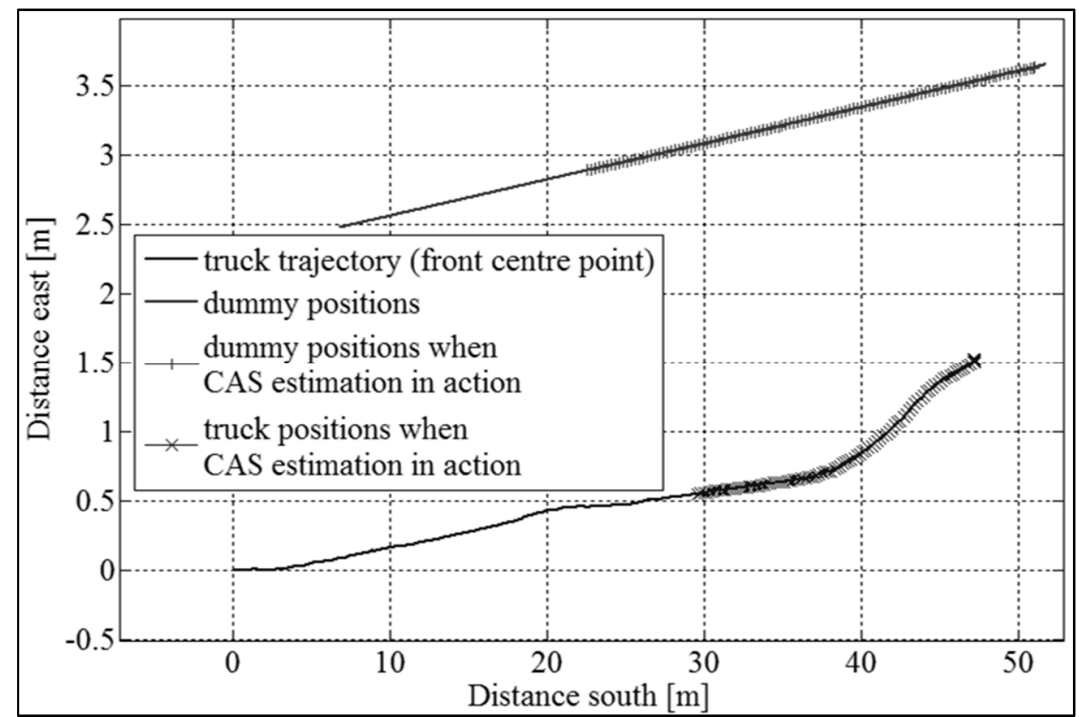

Fig. 10. A left turning trucking $(5 \mathrm{~km} / \mathrm{h})$ with a straight moving cyclist $(7 \mathrm{~km} / \mathrm{h})$ with trajectories displayed in the global coordinate system 


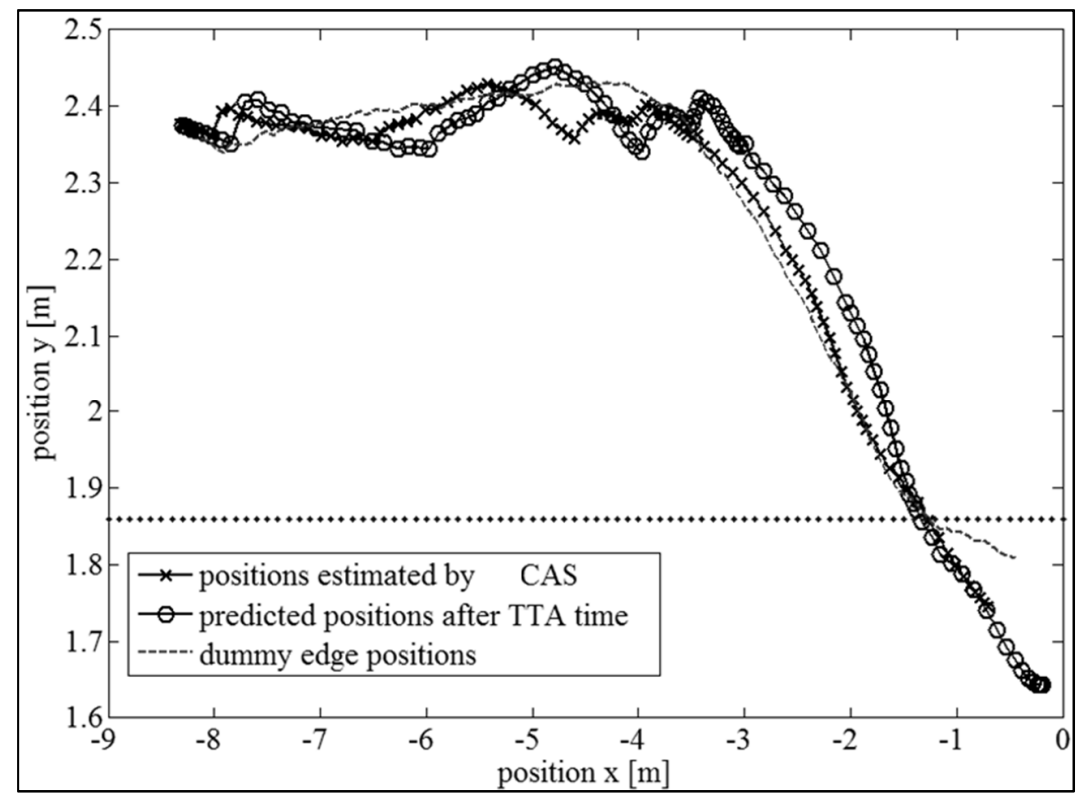

Fig. 11. Comparison of the estimated positions and the predicted positions after the TTA time

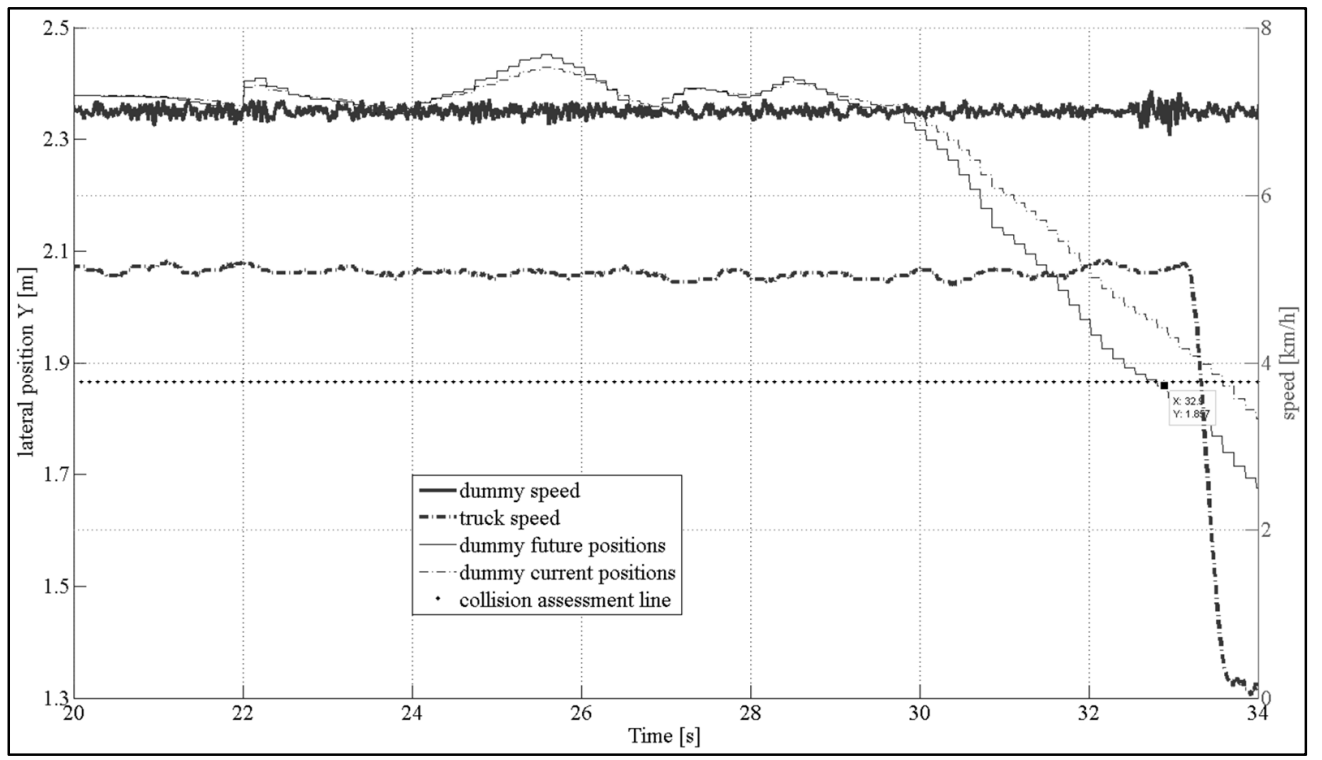

Fig. 12. Speed profiles of the dummy and the truck against the current and the predicted positions of the dummy 


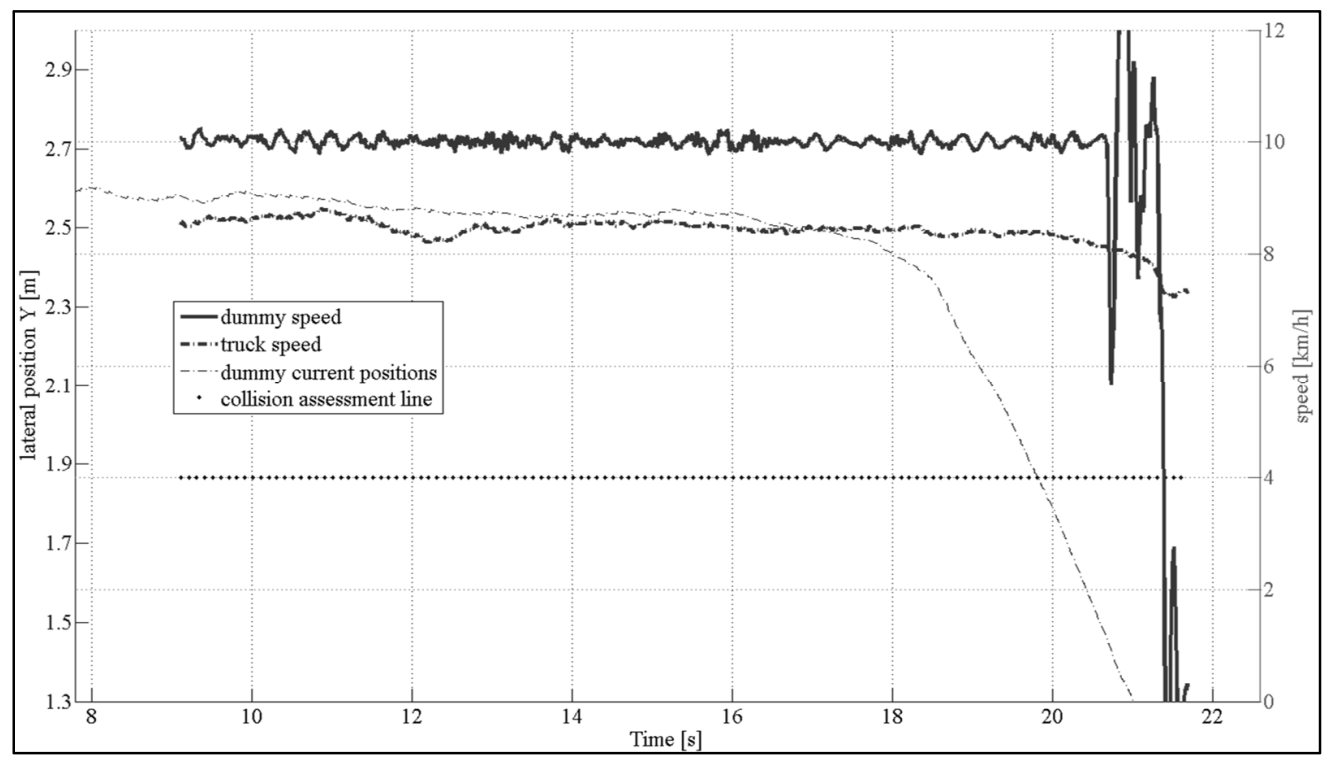

Fig. 13. Speed comparison for a test run with CAS disabled

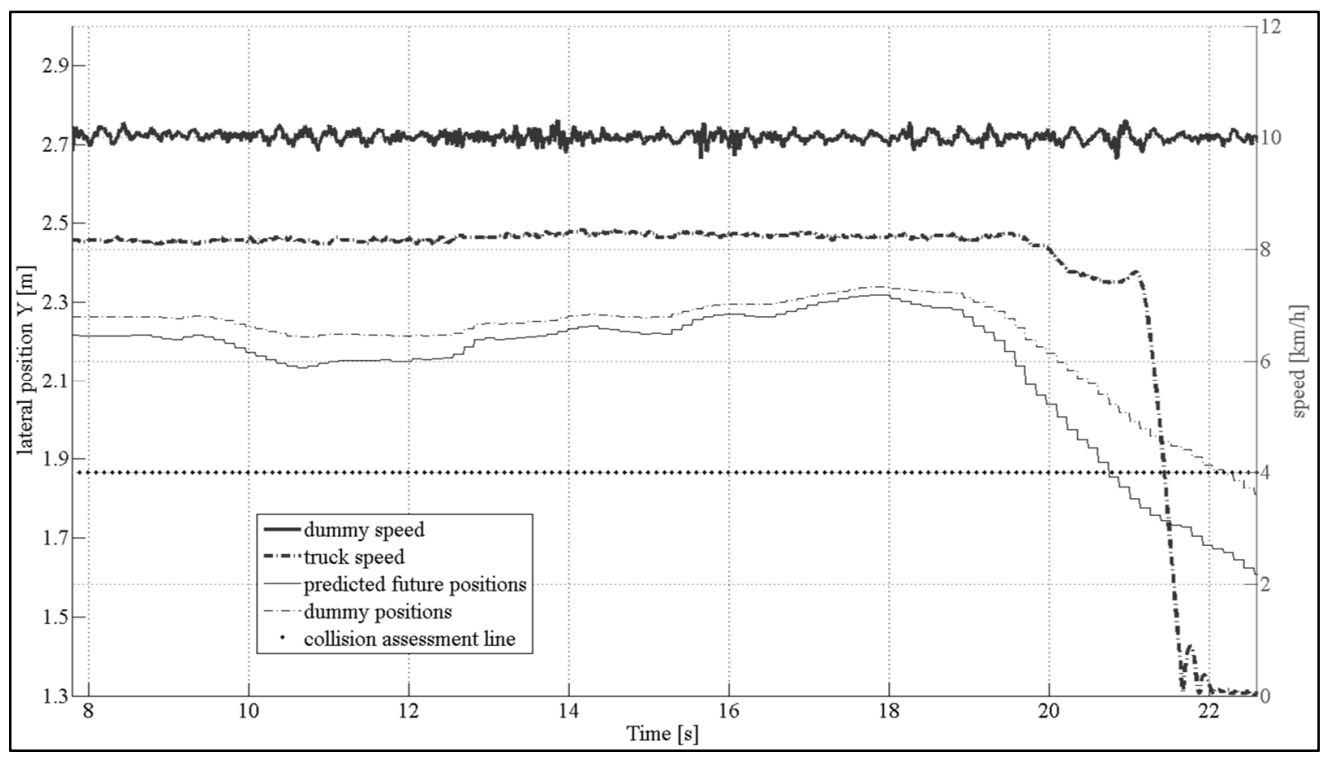

Fig. 14. Speed comparison for a test run with CAS enabled

\section{REFERENCES}

[1] Anonymous, "Transport Statistics Bulletin Road Freight Statistics 2009," Department for Transport, London, UK2009.

[2] Anonymous, "Consultation on the possibility of allowing an increase in the length of articulated lorries," Royal Society for the Prevention of Accidents, London, UK2011.

[3] Anonymous, "Cyclists and lorries fact sheet," Royal Society for the Prevention of Accidents, London, UK2006.

[4] E. Delmonte, et al., "Construction logistics and cyclist safety Technical report," 16/01/2013.

[5] Y. Jia and D. Cebon, "A strategy for Avoiding Collisions between Heavy Goods Vehicles," submitted to Proceedings of the Institution of Mechanical Engineers, Part C: Journal of Mechanical Engineering Science, 2015.

[6] Y. Jia, "An Automated Cyclist Collision Avoidance System for Heavy Goods Vehicles," Ph.D. Ph.D. thesis, Department of Engineering, University of Cambridge, Cambridge, 2014.

[7] Volvo. (2013). Volvo Car Group introduces world-first Cyclist Detection with full auto brake Available: https://www.media.volvocars.com/global/enhanced/en$\mathrm{gb} / \mathrm{media} /$ preview .aspx? mediaid $=48277$

[8] Y. Jia and D. Cebon, "Measuring motion of vulnerable road users relative to moving HGVs," submitted to IEEE Transactions onIntelligent Transportation Systems , 2015.

[9] P. J. Mosterman, et al., Embedded Real-Time Control via MATLAB, Simulink, and XPC Target, 2005.

[10] Anthony Best Dynamics, "Soft pedestrian target outline specification," A. B. D. Ltd, Ed., ed. Bradford-on-Avon, UK, 2014, pp. 1-2.

[11] Anthony Best Dynamics, "Pedal Robots outline specification," A. B. D. Ltd, Ed., ed. Bradford-on-Avon, UK, 2014, p. 2. 


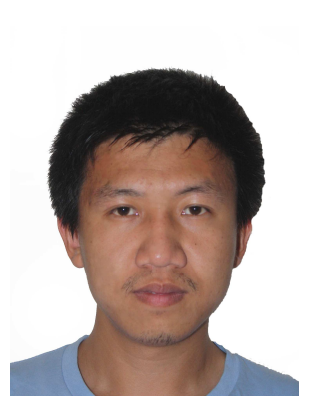

Yanbo Jia received the B.S. degree in Automotive Engineering from Jilin University, Changchun, Jilin Province, China, in 2009 and Ph.D. degree in Engineering in 2015 from University of Cambridge, Cambridge, UK.

He becomes the Research Associate in Cambridge University Engineering Department since March 2015. His research is focused on collision avoidance between cyclists and heavy goods vehicles, which covers sensors, signal processing, algorithm development, real-time system design and product prototyping.

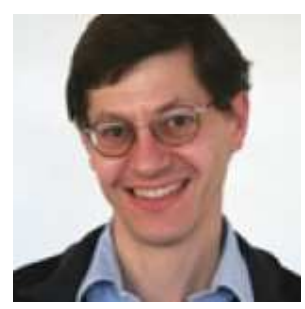

David Cebon received $\mathrm{BE}$ in mechanical engineering from University of Melbourne, Australia in
1980 and $\mathrm{PhD}$ degree in engineering from University of Cambridge, UK, in 1985.

He has been employed by the University of Cambridge since 1985 , first as a lecturer and more recently as professor of mechanical engineering. His research covers the mechanical, civil, and materials aspects of road transport engineering. He has authored or co-authored more than 150 papers on dynamic loads of heavy vehicles, road and bridge response and damage, advanced suspension design for heavy vehicles, heavy vehicle safety, mobility and fuel consumption and the micromechanics of asphalt deformation and fracture.

Prof. Cebon is a Fellow of the Royal Academy of Engineering and the Institution of Mechanical Engineers. He is Director of the Cambridge Vehicle Dynamics Consortium and the Centre for Sustainable Road Freight and he leads Cambridge University Engineering Department's Transport Research Group and the Engineering Department's research theme 'Energy, Transport and Urban Infrastructure'. He serves on the Editorial Boards of three international journals. 